\title{
Imaging characteristics and pathogenesis of intracranial artery stenosis in patients with acute cerebral infarction
}

\author{
WENYUAN XU, NING XIE, CHENG ZHANG and QIN HUANG \\ Department of Neurology, The First Affiliated Hospital of Nanchang University, Nanchang, Jiangxi 330006, P.R. China
}

Received June 4, 2017; Accepted August 1, 2017

DOI: $10.3892 /$ etm.2018.5965

\begin{abstract}
The current study aimed to investigate the imaging characteristics and pathogenesis of intracranial artery stenosis in patients with acute cerebral infarction. In total, 84 patients diagnosed with acute cerebral infarction were recruited. Magnetic resonance angiography was performed to detect the existence of intracranial artery stenosis or occlusion. In addition, magnetic resonance imaging and diffusion weighted imaging were employed to analyze the infarction types and characteristics. In the majority of patients, the infarction resulted from internal carotid stenosis (77 cases; 91.7\%), while it was caused by vertebral artery stenosis in a small number of cases (7 cases; 8.3\%). Multiple infarction was identified the most common type of infarction among all cases $(69.0 \%)$. The most common types of infarctions in the internal carotid system were multiple infarction implicating both the cortex and centrum ovale $(23.4 \%)$, and internal watershed infarction $(22.1 \%)$. Although the number of cases was relatively small, multiple infarction was observed to have a high incidence in the vertebral artery system. Bedside electrocardiogram was also recorded to determine the sinus rhythm and examine the abnormal hemodynamics. The sinus bradycardia rate of patients with multiple infarction was markedly greater in comparison with that in single infarction patients $\left(\chi^{2}=0.01\right.$, $\mathrm{P}<0.05)$. Transcranial Doppler plus microembolus monitoring was utilized to explore the possible pathogenesis of all types of infarctions, such as arterial embolization. As compared with the single infarction patients, the embolus rate in patients with multiple infarction was notably increased by $\sim 3$.7-fold $\left(\chi^{2}=8.65, \mathrm{P}<0.05\right)$. In conclusion, the cerebral infarction was common in the internal carotid system, with multiple infarction observed in the majority of cases. The pathogenesis of cerebral infarction included arterial embolization and inadequate hemoperfusion.
\end{abstract}

Correspondence to: $\mathrm{Dr}$ Wenyuan $\mathrm{Xu}$, Department of Neurology, The First Affiliated Hospital of Nanchang University, 17 Yongwaizheng Road, Nanchang, Jiangxi 330006, P.R. China E-mail: xuwenyuan01@163.com

Key words: intracranial artery stenosis, cerebral infarction, imaging, pathogenesis, embolization, hemoperfusion

\section{Introduction}

Cerebrovascular disease is one of the three most common causes of human mortality, with irreversible sequelae of severe disability reported in 50-70\% of surviving patients (1). The Chinese diagnostic guidelines of cerebrovascular diseases in 2010 stated that $7-10 \%$ of males and $5-7 \%$ of females aged $>65$ years presented carotid artery stenosis of $>50 \%$ (2). A retrospective study of symptomatic carotid artery stenosis and endometrectomy from North America revealed that the annual incidence of stroke in patients with 60-99\% stenosis was 3.2\% at a 5-year follow-up (3). In patients with $60-74 \%$ stenosis, the annual incidence of ipsilateral stroke was $3.0 \%$, while this incidence was elevated to $3.7 \%$ in patients with $75-94 \%$ stenosis (4).

Intracranial atherosclerotic stenosis is the most common cause of ischemic stroke worldwide and also results in the recurrence of stroke (5). Therefore, a complete and systematic understanding of the imaging characteristics and pathogenesis of intracranial artery stenosis will assist clinical experts in employing more efficient strategies to prevent and alleviate the induced clinical effects. An increasing number of modern imaging techniques (6), including magnetic resonance angiography (MRA), computed tomography angiography, digital subtraction angiography (DSA), transcranial Doppler (TCD), magnetic resonance imaging (MRI), diffusion weighted imaging (DWI) and microembolus (MES) monitoring, are developed to facilitate the inspection of intracranial artery stenosis and the examination of possible pathogenesis of the resulted cerebral infarction. Although DSA is the golden standard of determining the degree of vascular stenosis, accurate measurement is dependent on the rapid injection of a large dosage of contrast agents into the artery, which may induce allergenic reactions (7). Furthermore, it is an expensive and invasive technique (7). Non-invasive tools, including MRA and TCD, can be used to detect moderate to severe stenosis (50-99\%) as reliable methods (8). MRA is the most common strategy for the diagnosis of intracranial artery stenosis due to its accuracy, inexpensiveness, impersonality, intuition and non-invasion. It is also convenient in achieving doctor-patient communication and provides the required conditions for further DSA examination (9). DWI is able to diagnose minimal infarction lesions in the cortex and periventricular regions, but also to discover ultra-early ischemic alterations in brain tissues and definitely demonstrate the new-onset infarction lesions (10). 
Therefore, DWI has become a favorable method used for the investigation of the imaging characteristics of infarction (10). Furthermore, TCD plus MES monitoring is conducive to the detection of intracranial embolismic diseases (10).

In the present study, the imaging characteristics and pathogenesis of intracranial artery stenosis were investigated in patients with acute cerebral infarction using various modern imaging tools. DWI was employed to analyze the lesion characteristics of intracranial artery stenosis in these patients in detail. Furthermore, TCD, MES monitoring and electrocardiogram (ECG) alteration techniques were combined to investigate the pathogenesis of acute cerebral infarction induced by intracranial artery stenosis.

\section{Materials and methods}

Subjects. A total of 84 cases of patients undergoing acute cerebral infarction during the week prior to admission between October 2008 and June 2012 in the Department of Neurology at the First Affiliated Hospital of Nanchang University (Nanchang, China) were recruited into the current study, subsequent to signing informed consent forms. The study protocol was reviewed and approved by the Institutional Ethics Committee of the First Affiliated Hospital of Nanchang University. The cohort was comprised of 74 males and 10 females with an age range of 41-80 years and a mean age of $61 \pm 9$ years. All patients were diagnosed according to the diagnostic criteria on cerebrovascular diseases revised by the Fourth Chinese Academic Conference (11). The existences of intracranial artery stenosis or occlusion, including 77 cases of anterior circulation artery occlusion (internal carotid artery) and 7 cases of posterior circulation artery occlusion (vertebrobasilar artery), were diagnosed according to MRA findings. Patients with cardioembolism, arteritis, syphilis, concurrent infection, cancer or severe cardiopulmonary diseases were excluded.

Imaging data. Upon admission all patients were examined by MRI (Trio3.0T; Siemens AG, Munich, Germany) using the axial T1-weighted image (T1WI), T2WI, DWI, sagittal T2WI and fluid-attenuated inversion recovery sequences. DWI was employed to analyze the morphological features of infarction. Bright hyperintensity displayed on DWI indicated a fresh infarct lesion. Any observed changes, which indicated infarction were confirmed by an MRI radiologist and two senior neurologists.

Infarct location and lesion morphology. The cases included in the current study included internal carotid artery stenosis and vertebrobasilar artery stenosis patients. On the basis of regional division, internal carotid artery infarctions were divided into cortex infarction, subcortex infarction, complete infarction, incomplete infarction that simultaneously implicated the cortex and subcortex, deep infarction and watershed infarction. Similarly, vertebrobasilar artery infarctions were divided into cortex infarction, deep infarction, and infarction that implicated the cortex and deep vessels. In terms of the morphology distribution, internal carotid artery and vertebrobasilar artery infarction was recognized as single or multiple infarction (two or more infarction lesions in the same or various locations). Regarding the infarct size, lesions were divided into the small (maximal diameter $<20 \mathrm{~mm}$ ), moderate (20 $\mathrm{mm}<$ maximal diameter $<40 \mathrm{~mm}$ ) and large infarction (maximal diameter $>40 \mathrm{~mm}$ ).

Sinus bradycardia assessment. All patients received complete bedside ECG examination within $48 \mathrm{~h}$ after hospitalization. Sinus bradycardia in the patients was defined as a ventricular rate of $<60$ beats/min on the ECG.

TCD and MES monitoring. All patients underwent TCD with MES monitoring within 3 days after hospitalization at a frequency of one time per day. Related arteries were monitored repeatedly within a short time (<30 min) using TCD (Digi-Lite system; Rimed Ltd., Ra'anana, Israel). The TCD device also performed the MES monitoring. The embolus was observed by physicians using the TCD-8 software for Multi-DopX4, version 8.00Q (DWL, Sipplingen, Germany) and defined as either gaseous or solid. The examination results were confirmed by two professional TCD doctors.

Statistical analysis. A database was established with Excel 2003 (Microsoft Corp., Redmond, WA, USA) and then imported into the SAS version 9.2 software (SAS Institute Inc., Cary, NC, USA) for statistical analysis. Qualitative data were analyzed by $\chi^{2}$-test, while quantitative data were analyzed by student's t-test for comparison of two independent samples. A P-value of $<0.05$ was considered to indicate differences that were statistically significant.

\section{Results}

Infarction types and morphology. The data regarding the infarction types and morphology of all patients are shown in Table I and Fig. 1. Among the 84 patients, the majority of infarction cases resulted from internal carotid stenosis (77 patients; 91.7\%), while a small number of cases resulted from vertebral artery stenosis (7 patients; $8.3 \%$ ). However, the difference in the proportion of multiple infarctions between the internal carotid stenosis and vertebral artery stenosis was not statistically significant $\left(\chi^{2}=0.02, \mathrm{P}>0.05\right)$. Multiple infarction was observed to be the most common type of infarction in the two stenosis groups $(69.0 \%)$.

The specific infarction types and morphology in the 77 cases of infarction in the internal carotid system are demonstrated in Table II. Typical images of every infarction type in the internal carotid system are shown in Fig. 2. The results indicated that multiple infarction (implicating both the cortex and centrum ovale, $23.4 \%$ ) and internal watershed infarction (22.1\%) were the two most common types of infarction in the internal carotid system. Cortex large single infarction (3.9\%), centrum ovale single infarction $(2.6 \%)$, and anterior and posterior watershed infarction $(1.3 \%)$ were infrequent in internal carotid stenosis.

Table III depicts the specific infarction types and morphology in the 7 cases of infarction in the vertebral artery system. Typical images of each infarction type in the vertebral artery system are also shown in Fig. 3. Although the number of cases was relatively small, a high incidence of multiple infarction was also observed in these patients. 
Table I. Infarction types and morphology in 84 cases.

Infarct morphology

\begin{tabular}{lccc} 
Infarction site & Cases $(\%)$ & Multiple infarction (\%) & Single infarction (\%) \\
\hline Internal carotid system & $77(91.7)$ & $53(63.0)$ & $24(28.0)$ \\
Vertebral artery system & $7(8.3)$ & $5(6.0)$ & $2(3.0)$ \\
Total & 84 & $58(69.0)$ & $26(31.0)$ \\
\hline
\end{tabular}

Table II. Infarction types and morphology in 77 cases of infarction in the internal carotid system.

\begin{tabular}{lc}
\hline Infarction types and morphology & Cases, $\mathrm{n}(\%)$ \\
\hline Complete infarction & $9(11.7)$ \\
Cortex infarction & \\
Multiple & $8(10.3)$ \\
Large, single & $3(3.9)$ \\
Centrum ovale infarction & \\
Single & $2(2.6)$ \\
Medullary multiple & $9(11.7)$ \\
Multiple infarction & \\
Deep small infarction & $18(23.4)$ \\
Watershed infarction & $10(13.0)$ \\
Anterior and posterior & \\
Internal & $1(1.3)$ \\
\hline
\end{tabular}

amplicating the cortex and centrum ovale.

Sinus bradycardia in different infarction patterns. The sinus rhythm in patients with different infarction patterns is demonstrated in Table IV. It was observed that the sinus bradycardia rate of patients with multiple infarction $(31.0 \%)$ was markedly greater in comparison with that in patients with single infarction $\left(11.5 \% ; \chi^{2}=0.01, \mathrm{P}<0.05\right)$. The sinus bradycardia rate in all patients was $25.0 \%$.

Embolus in different infarction patterns. The incidence of embolus in patients with various patterns of infarction is listed in Table V. The results identified that, as compared with patients with single infarction $(7.7 \%)$, the embolus rate in patients with multiple infarction was notably increased to $\sim 4.7$-fold $\left(36.2 \% ; \chi^{2}=8.65, \mathrm{P}<0.05\right)$. The embolus rate in all patients was $27.4 \%$.

\section{Discussion}

The imaging features of cerebral infarction caused by intracranial artery stenosis have been reported in the literature, however, detailed studies are lacking. In the study by Chen et al (12), a total of 62 patients were recruited with acute cerebral infarction who met the following criteria: i) The location of acute infarction was confirmed by DWI within 1 week

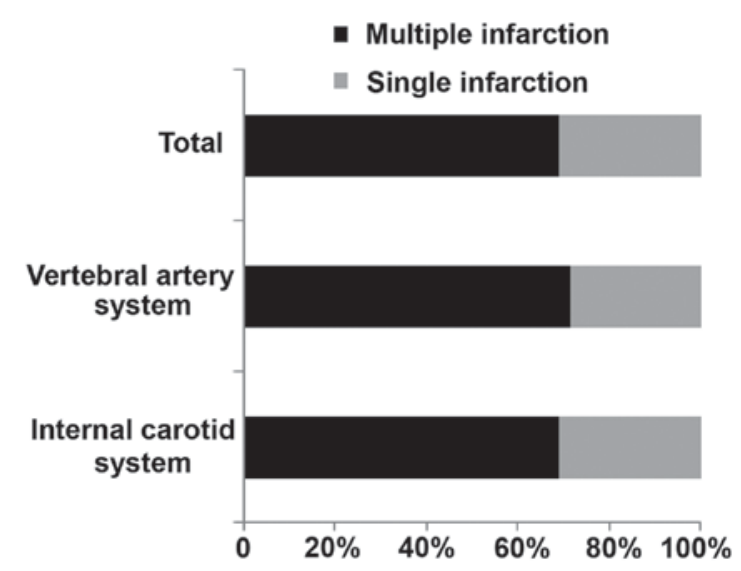

Figure 1. Distribution of infarct size in 84 patients with different types of arterial stenosis.

after the onset of disease; ii) the lesion was located in the intracranial segment of the carotid artery; and iii) the infarction was identified to be induced by the ipsilateral carotid artery occlusion rather than other causes. According to the distribution of DWI lesions in these patients, the infarct types were classified as perforating artery infarct (PAI), pial infarct (PI), border-zone infarct (BZI) and multiple infarcts, indicating a combination of the aforementioned types (12). In addition, Kang et al (13) employed DWI to survey the infarction types of 35 patients with acute cerebral infarction induced by internal carotid artery occlusion. The study identified 21 cases of regional infarction (non-watershed infarction), including 9 cases of cortex multiple infarction, 7 cases of cortex and deep basal ganglia region multiple infarction, and 5 cases of single infarction in these sites (13). Others types included 9 cases of watershed infarction combined with the regional infarction, 1 case of simple watershed infarction and 4 cases of bilateral cerebral hemisphere infarction.

Tatu et al (14) reported the criteria for evaluating the infarction of the middle cerebral artery (MCA) region. According to the infarct site, volume and distribution demonstrated on DWI, the cerebral infarction was divided into cortex regional infarction, deep small infarction, watershed infarction and multiple infarction (14). The cortex regional infarction lesion was located in the main branch of MCA, cortical or medullary blood supply area, and the cortex regional infarction included the MCA complete, MCA cortical branch, small cortical and centrum ovale infarctions. The infarction in patients with MCA occlusive disease (MCAOD) was presented by imaging as multiple 
A

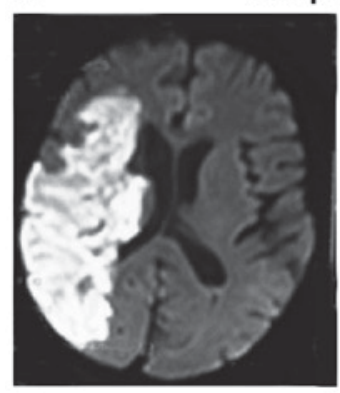

C

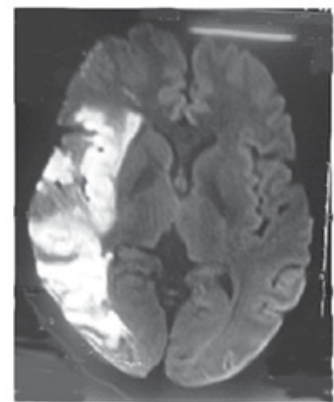

Medullary multiple infarction

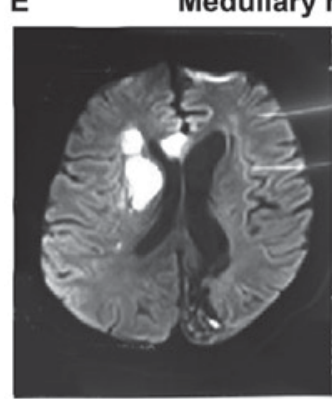

G

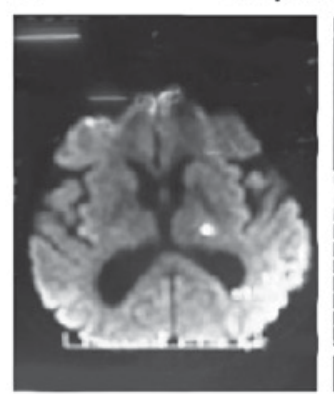

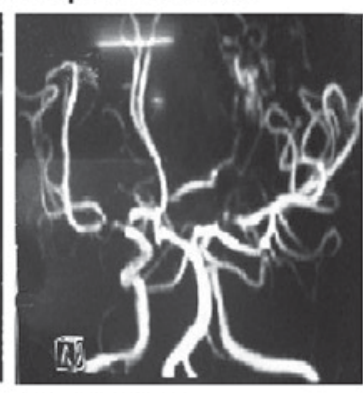

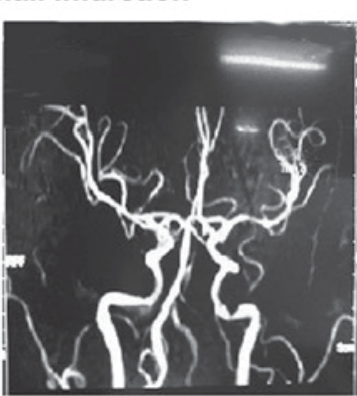

B

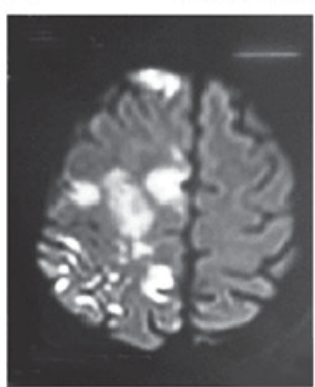

Cortex multiple infarction

D

Centrum ovale single infarction
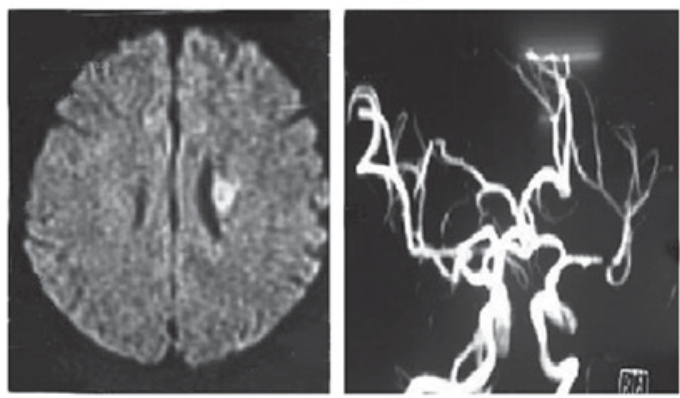

F Cortex and centrum ovale multiple infarction
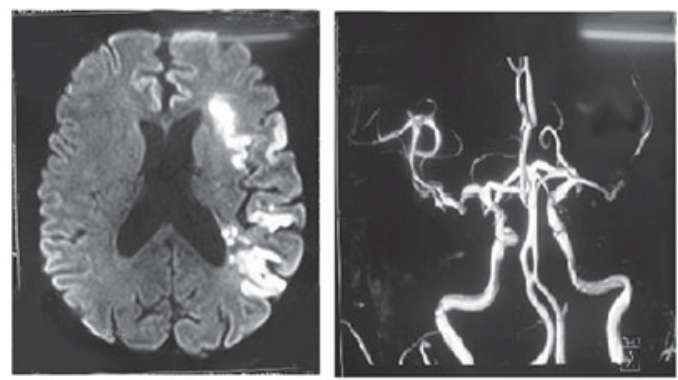

H

Watershed infarction
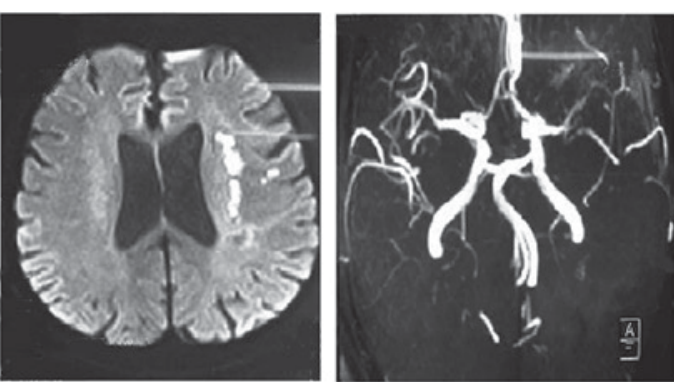

Figure 2. Typical images of different infarction types in the internal carotid system. For each type, DWI scans are shown on the left, and magnetic resonance imaging scans are shown on the right. (A) Complete infarction, demonstrated by the occlusion of the right internal carotid artery and right MCA (right image), and complete infarction of the cortex and perforating branch in the blood supply area of the right MCA (larger area infarction) in DWI scan (left image). (B) Cortex multiple infarction, where the occlusion of bilateral MCA and right anterior cerebral artery is observed (right image), and DWI demonstrates multiple cerebral infarction with nonuniform sizes of infarction lesions and various shapes of round, oval and large nodules in the right parietal cortex (left image). (C) Cortex large single infarction, with occlusion of the right MCA (right image), and DWI demonstrating the cortex infarction in the blood supply area of the right MCA (left image). (D) Centrum ovale single infarction, with local stenosis of the left MCA (right image), and the centrum ovale single infarction observed in DWI (left image). (E) Medullary multiple infarction, with local stenosis of bilateral MCA (right image), and multiple infarction in the right medullary area observed in DWI (left image). (F) Cortex and centrum ovale multiple infarction, with local stenosis of left MCA (right image), and DWI showing the multiple infarction in the cortex and the centrum ovale (left image). (G) Deep small infarction, with local stenosis of left MCA (right image), and DWI showing the lacunar infarction in the basal ganglia region (left image). (H) Watershed infarction, with stenosis of left MCA (right image), and DWI showing the internal watershed infarction with bunchy infarction lesions around the left lateral ventricle (left image). DWI, diffusion weighted imaging; MCA, middle cerebral artery.

small cortical infarction, which was often accompanied with centrum ovale infarction (15). In the lenticulostriate artery region, deep small infarction was defined as 'giant lacuna' with a lesion diameter of $>15 \mathrm{~mm}$, striatocapsular infarction and lacunar infarction (16). Of the MCA lesions,
$30.7 \%$ presented lacunar infarction, while lacunar and striatocapsular infarctions were the most common types of basal ganglionic infarction (16). A study focusing on the Chinese and Korean populations also pointed out that the majority of patients bearing lacunar or deep striatocapsular infarction 
Table III. Infarction types and morphology in 7 cases of infarction in the vertebral artery system.

\begin{tabular}{lc} 
Infarction types and morphology & Cases, $\mathrm{n}(\%)$ \\
\hline Deep single infarction & $1(14.3)$ \\
Cortical single infarction & $1(14.3)$ \\
Multiple infarction & $5(71.4)$
\end{tabular}

Table IV. Sinus rhythm in patients with different infarction patterns.

\begin{tabular}{lccc}
\hline & & \multicolumn{2}{c}{ Sinus bradycardia } \\
\cline { 3 - 4 } $\begin{array}{l}\text { Infarct } \\
\text { morphology }\end{array}$ & $\begin{array}{c}\text { Infarction } \\
\text { cases, } \mathrm{n}\end{array}$ & $\begin{array}{c}\text { Cases, } \\
\mathrm{n}\end{array}$ & $\begin{array}{c}\text { Rate, } \\
\%\end{array}$ \\
\hline $\begin{array}{l}\text { Multiple infarction } \\
\text { Single infarction }\end{array}$ & 58 & 18 & 31.0 \\
Total & 26 & 3 & 11.5 \\
\hline
\end{tabular}

Table V. Embolus in patients with different infarction patterns.

\begin{tabular}{lccc}
\hline $\begin{array}{l}\text { Infarct } \\
\text { morphology }\end{array}$ & $\begin{array}{c}\text { Infarction } \\
\text { cases, } \mathrm{n}\end{array}$ & $\begin{array}{c}\text { Embolus } \\
\text { cases, } \mathrm{n}\end{array}$ & $\begin{array}{c}\text { Embolus } \\
\text { rate, \% }\end{array}$ \\
\hline Multiple infarction & 58 & 21 & 36.2 \\
Single infarction & 26 & 2 & 7.7 \\
Total & 84 & 23 & 27.4 \\
\hline
\end{tabular}

had occlusive lesions in the M1 segment of the ipsilateral MCA or internal carotid artery (17).

Watershed infarction includes the anterior, posterior and internal watershed infarcts. In recent years, cerebral watershed infarction has gradually become an important infarct type of MCAOD. Wong et al has also reported that chain-type watershed infarction was the most common type in patients with acute cerebral infarction resulting from MCA occlusion (18). Watershed infarction has been identified to be common in severe stenosis or occlusion of the internal carotid artery (19). Furthermore, progressive aggravation of neurological impairment in patients with subcortical cerebral infarction is associated with the atherosclerotic stenosis of the corresponding arteries; thus, intracranial artery stenosis serves an important role in the neurological deterioration of patients with subcortical infarction (20).

Multiple infarction refers to the multiple and discontinuous non-fused infarction lesion in MCA region. In a previous study conducted at the Chinese University of Hong Kong investigated 30 cases of acute stroke by DWI (18), demonstrating that $50 \%$ of patients presented multiple infarction, where the cortical multiple infarction accounted for $60 \%$; PAI patients accounted for $66.7 \%$ of cases, with $2 / 3$ presenting multiple lesions. In addition, $73 \%$ of patients suffered watershed
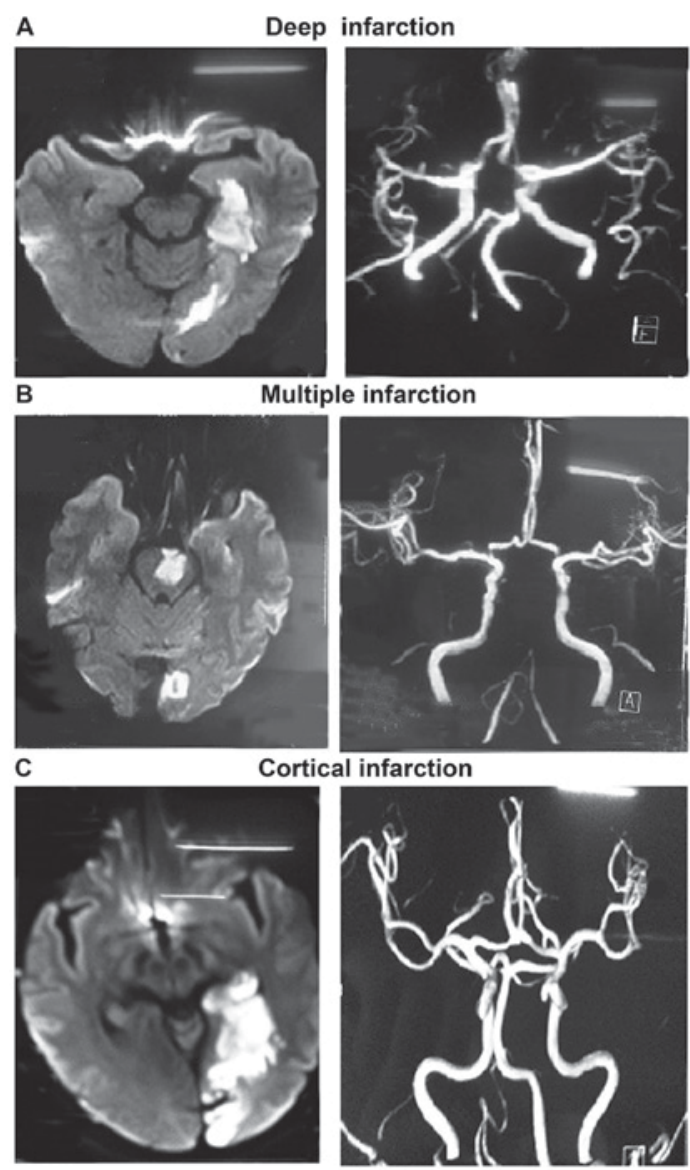

Figure 3. Typical images of each infarction type in the vertebral artery system. For each type, DWI scans are shown on the left, and magnetic resonance imaging scans are shown on the right. (A) Deep infarction, with occlusion of the left posterior cerebral artery (right image), and DWI showing the internal infarction of the temporal and occipital lobes (left image). (B) Multiple infarction, with occlusion of the basilar artery (right image), and DWI showing the multiple infarction in the blood supply area of the posterior circulation (pons and occipital lobe; left image). (C) Cortical infarction, with occlusion of the left vertebral artery (right image), and DWI showing the cortex infarction in the left occipital lobe (left image). DWI, diffusion weighted imaging.

infarction, and three types of watershed infarction in the anterior circulation were identified in MCAOD patients, where the internal border zone was the most frequently affected site and multifocal distribution with an arrangement of chain-type was observed (21). Furthermore, the study by Tan and Yang (22) also reported that multiple infarction was the most common type in intracranial artery stenosis.

In agreement with the aforementioned studies, the present study revealed that the incidence of cerebral infarction, including multiple and single infarctions, was evidently higher in the internal carotid system rather than the vertebral artery system, while multiple infarction $(69.0 \%)$ was also much more frequent in comparison with single infarction (31.0\%) in the two artery systems. In particular, multiple infarction implicating both the cortex and centrum ovale (23.4\%) and internal watershed infarction (22.1\%) were the two infarction types with the highest incidence. Since the literature regarding the imaging characteristics of cerebral infarction induced by posterior circulation artery stenosis is limited, the present study enriched and deepened the knowledge on these features. 
However, in comparison with the internal carotid system, the cases involving the vertebral artery system were significantly lower. Thus, a large-scale investigation on vertebral artery stenosis should be performed in the future to obtain more abundant clinical evidence. Furthermore, the infarction types in carotid artery stenosis reported herein were different from the previous classification (12). The classification in the present study was considered to be more appropriate and specific compared with the previous classification.

In a previous study, it was confirmed that the constituents (fibrins and platelet aggregates) of thromboembolism causing occlusion of the distal artery in patients with internal carotid artery stenosis were the same as those of the surface thrombus of atherosclerotic plaques in terms of pathology; therefore, a mechanism of arterial embolization was proposed (23). Kang et al (13) considered that the acute cerebral infarction induced by the occlusion of the internal carotid artery was multiple infarction, and that arterial embolization was the leading cause of internal carotid artery stroke. Gao et al (24) also reported that intracranial multiple lesions observed on DWI scans often suggested the possible pathogenesis of embolism. Furthermore, a previous TCD study reported that MES signals were more likely to be detected in patients with intracranial artery stenosis that had multiple lesions (25). In the present study based on 84 patients with intracranial artery stenosis and cerebral infarction, it was revealed that the embolus cases in multiple infarction were notably greater in comparison with those in single infarction. It was indicated that multiple infarction mainly resulted from embolization, which was in agreement with the aforementioned previous studies. Thus, arterial embolization can be considered as a principle cause of multiple cerebral infarction.

Chen et al (12) investigated the infarction types and pathogenesis in 62 patients with intracranial artery stenosis and observed that hypoperfusion was the main cause of watershed infarction (12). The corresponding imaging finding was subcortical white matter with internal chain-typed distribution or low-density lesion with striped distribution (26). In the current study, watershed infarction with a significant majority of internal watershed infarction accounted for nearly one third of the 58 cases of multiple infarction. Furthermore, ECG demonstrated that the incidence of sinus bradycardia in patients with multiple infarction was significantly higher compared with that in patients with single infarction; this observation has not been reported in previous publications, to the best of our knowledge. Thus, it was speculated that cerebral hypoperfusion induced by bradycardia may be an important pathogenesis mechanism of watershed infarction or multiple infarction. Watershed infarcts were usually confluent and MES was more likely to appear, suggesting that watershed infarction may be the result of decreased embolus clearance caused by hypoperfusion in this region (18).

Apart from the multiple infarction, 12 cases of single infarction in the medullary area and lacunar infarction in the basal ganglia region were recruited in the present study. In these patients, the positive rate of embolus and the incidence of sinus bradycardia on ECG were low. The pathogenesis of single infarction in the medullary area and lacunar infarction in the basal ganglia region may be a result of thrombosis induced by de novo vascular impairment, such as atherosclerosis. Thrombosis is known as an important pathogenesis of cerebral infarction (27). For instance, the thrombosis in MCAOD patients may be associated with the generation of deep small infarction (28). In particular, stenosis in the main branch of the MCA may result in thrombosis in the plaque residues and implicate the ostia of the perforating artery, thereafter inducing the deep small infarction in MCAOD patients (28). The single infarction within the cortex region was also reported to be connected with the thrombosis of the perforating artery (18).

In conclusion, the present study observed that cerebral infarction, including multiple and single infarctions, was evidently more common in the internal carotid system as compared with the vertebral artery system, with multiple infarction observed in the majority of cases in the two artery systems. In particular, multiple infarction implicating the cortex and centrum ovale, and internal watershed infarction were the most common types. The pathogenesis of cerebral infarction due to intracranial artery stenosis included arterial embolization and inadequate hemoperfusion. Furthermore, sinus bradycardia served an important role in the generation of cerebral infarction by hypoperfusion.

\section{Competing interests}

The authors declare that they have no competing interests.

\section{References}

1. Ahmed Ashrafi SK, Suhail Z and Khambaty Y: Postembolization infarction in juvenile nasopharyngeal angiofibroma. J Coll Physicians Surg Pak 21: 115-116, 2011.

2. Guidelines for secondary prevention of ischemic stroke and transient ischemic attack in China 2010. Chin J Neurol 43: 154-160, 2010 (In Chinese).

3. Paciaroni M, Eliasziw M, Kappelle LJ, Finan JW, Ferguson GG and Barnett HJ: Medical complications associated with carotid endarterectomy. North American Symptomatic Carotid Endarterectomy Trial (NASCET). Stroke 30: 1759-1763, 1999.

4. Ferguson GG, Eliasziw M, Barr HW, Clagett GP, Barnes RW, Wallace MC, Taylor DW, Haynes RB, Finan JW, Hachinski VC and Barnett HJ: The North American symptomatic carotid endarterectomy Trial: Surgical results in 1415 patients. Stroke 30: $1751-1758,1999$.

5. Koo J: The latest information on intracranial atherosclerosis: Diagnosis and treatment. Interv Neurol 4: 48-50, 2015.

6. Yin P, Feng JC and Wang SC: Pathogenesis of cerebral watershed infarction and the compensatory effect of colleteral ability of the Willis circle. Chin J Cerebrovasc Dis 5: 102-106, 2008 (In Chinese).

7. Ma G, Jiang Z, He J, Liao Y, Zhu M, Huang Z and Cui F: The value of DSA and CTA for diagnosis of carotid artery stenosis. Clin Med Eng 22: 535-536, 2015 (In Chinese).

8. Khan M, Naqri L, Bansari A and Kamal AK: Intracranial atherosclerotic disease. Stroke Res Treat 2011: 282845, 2011.

9. Deng X, Liu W, Liu J and Zhong L: A study of MRA for the diagnosis of occlusive and stenotic disorders of intracranial arteries in ischemic cerebral vascular disease. J Pract Med Tech 14: 1968-1970, 2007.

10. Qiu CC, Lu H, Wu Q and Yu JY: Retrospective analysis of clinical value of early diagnosis in brainstem infarction by magnetic resonance imaging characteristics with MRI and DWI. Chin J Trauma Disabil Med 21: 22-24, 2013 (In Chinese).

11. $\mathrm{Wu} \mathrm{CH}$ and Liu B: The fourth Chinese academic conference on cerebrovascular disease. Chin Med News: 4, 1996.

12. Chen H, Hong H, Liu D, Xu G, Wang Y, Zeng J, Zhang R and Liu X: Lesion patterns and mechanism of cerebral infarction caused by severe atherosclerotic intracranial internal carotid artery stenosis. J Neurol Sci 307: 79-85, 2011. 
13. Kang DW, Chu K, Ko SB, Kwon SJ, Yoon BW and Roh JK: Lesion patterns and mechanism of ischemia in internal carotid artery disease: A diffusion-weighted imaging study. Arch Neurol 59: 1577-1582, 2002.

14. Tatu L, Moulin T, Bogousslavsky J and Duvernoy H: Arterial territories of the human brain: Cerebral hemispheres. Neurology 50: 1699-1708, 1998.

15. Liu JY, Wei JH and Wang JR: Stroke pattern analysis in patients with middle cerebral artery occlusive disease. J Apoplexy Nery Dis 22: 246-247, 2005.

16. Niizuma K, Shimizu H, Takada S and Tominaga T: Middle cerebral artery plaque imaging using 3-Tesla high-resolution MRI. J Clin Neurosci 15: 1137-1141, 2008.

17. Bang OY, Heo JH, Kim JY, Park JH and Huh K: Middle cerebral artery stenosis is a major clinical determinant in striatocapsular small, deep infarction. Arch Neurol 59: 259-263, 2002.

18. Wong KS, Gao S, Chan YL, Hansberg T, Lam WW, Droste DW, Kay R and Ringelstein EB: Mechanisms of acute cerebral infarctions in patients with middle cerebral artery stenosis: A diffusion-weighted imaging and microemboli monitoring study. Ann Neurol 52: 74-81, 2002.

19. Li HF, Zhang X, Zhang Y, Pan XD, Zhao HQ and Li H: Clinical and neuroradiological features of internal watershed infarction and the occlusive diseases of carotid artery system. Neurol Res 32: 1090-1096, 2010.

20. Hallevi H, Chernyshev OY, EI khoury R, Soileau MJ, Walker KC, Grotta JC and Savitz SI: Intracranial atherosclerosis is associated with progression of neurological deficit in subcortical stroke. Cerebrovasc Dis 33: 64-68, 2012.

21. Qiao Y, Liu L and Dong ZJ: The subtype of watershed infarction and the internalcarotid artery/middle cerebral artery steno-occlusion. Inner Mongolia Med J 46: 1427-1429, 2014.
22. Tan $\mathrm{H}$ and Yang Zhi: Types of infarction in patients with different degrees of middle cerebral artery stenosis and occlusion. Chin J Nervous Mental Dis 36: 427-429, 2010.

23. Masuda J: A pathologic study of carotid artery disease as an embolicsource. Jpn J Stroke 23: 347-350, 2001.

24. Gao S, Huang J, Huang Y and Li S: Infarction pathogenesis of atherosclerotic middle cerebral artery stenosis. Chin J Neurol 36: 155-157, 2003 (In Chinese).

25. Shi MC, Wang SC, Zhou HW, Xing YQ, Cheng YH, Feng JC and $\mathrm{Wu} \mathrm{J}$ : Compensatory remodeling in symptomatic middle cerebral atherosclerotic stenosis: A high-resolution MRI and microemboli monitoring study. Neurol Res 34: 153-158, 2012.

26. Ryoo S, Park JH, Kim SJ, Kim GM, Chung CS, Lee KH, Kim JS and Bang OY: Branch occlusive disease: Clinical and magnetic resonance angiography findings. Neurology 78: 888-896, 2012.

27. Katakami N, Takahara M, Kaneto H, Shimizu I, Ohno K, Ishibashi F, Osonoi T, Kashiwagi A, Kawamori R, Shimomura I, et al: Accumulation of gene polymorphisms related to plaque disruption and thrombosis is associated with cerebral infarction in subjects with type 2 diabetes. Diabetes Care 33: 390-395, 2010

28. Caplan LR: Intracranial branch atheromatous disease: A neglected, understudied, and underused concept. Neurology 39 1246-1250, 1989. 